\title{
Heritability estimates for beta cell function and features of the insulin resistance syndrome in UK families with an increased susceptibility to Type 2 diabetes
}

\author{
G. W. Mills ${ }^{1,2}$ • P. J. Avery ${ }^{2}$ M. I. McCarthy ${ }^{3,4}$ • A. T. Hattersley ${ }^{5}$ J. C. Levy • G. A. Hitman L M. Sampson $^{7}$ • \\ M. Walker ${ }^{1}$ \\ ${ }^{1}$ School of Clinical Medical Sciences, 4th Floor William Leech Block, The Medical School, University of Newcastle upon Tyne, \\ Newcastle upon Tyne, UK \\ ${ }^{2}$ School of Mathematics and Statistics, University of Newcastle upon Tyne, Newcastle upon Tyne, UK \\ ${ }^{3}$ Wellcome Trust Centre for Human Genetics, University of Oxford, Oxford, UK \\ ${ }^{4}$ The Oxford Centre for Diabetes, Endocrinology and Metabolism, University of Oxford, Oxford, UK \\ ${ }^{5}$ Department of Diabetes and Vascular Medicine, University of Exeter, Exeter, UK \\ ${ }^{6}$ Centre for Diabetes and Metabolic Medicine, Barts and the London, Queen Mary's School of Medicine and Dentistry, \\ University of London, London, UK \\ ${ }^{7}$ Department of Diabetes and Endocrinology, Norfolk and Norwich University Hospital, Norwich, UK
}

\section{Abstract}

Aims/hypothesis. The aim of this study was to measure the heritability estimates for metabolic traits and the features of the insulin resistance syndrome in families with an increased genetic susceptibility to Type 2 diabetes.

Methods. A total of 811 non-diabetic relatives from 278 pedigrees of northern European extraction in which there was a sib-pair with Type 2 diabetes were recruited and studied at the six Diabetes UK Warren Type 2 diabetes centres. Heritability estimates were calculated, allowing for key covariates (age, sex, BMI and recruitment centre). Values greater than 0.10 were considered statistically significant in comparison to zero.

Results. Fasting glucose concentration and homeostasis model assessment of pancreatic beta cell function (HOMA \%B) had the highest heritability estimates of 0.72 and 0.78 respectively. Heritability estimates for the features of the insulin resistance syndrome (BMI, WHR, systolic and diastolic blood pressure, serum lipids and homeostasis model assessment of insulin sensitivity [HOMA \%S]) were also high. The heritability estimate for fasting glucose was markedly higher in the present study ( 0.77 vs 0.21 adjusted for age and sex; $p<0.001)$ than in a comparable study of families from the same background population but with no increased susceptibility to diabetes. However, the estimates for the features of the insulin resistance syndrome were similar in the two studies.

Conclusions/interpretation. In families with a high risk of Type 2 diabetes, the heritability estimates for fasting glucose, pancreatic beta cell function and the features of the insulin resistance syndrome were all high. The higher heritability estimate for pancreatic beta cell function suggests that this resource may be most effective when investigating genetic susceptibility to beta cell dysfunction.

Keywords Heritability - Quantitative traits ·

Risk of Type 2 diabetes - Insulin resistance syndrome · Genetic susceptibility
Abbreviations: HOMA \%B, homeostasis model assessment of beta cell function - $\mathrm{HOMA} \% \mathrm{~S}$, homeostasis model assessment of insulin sensitivity $\cdot$ EIR, early insulin response 


\section{Introduction}

Type 2 diabetes is characterised by impaired pancreatic beta cell function and insulin resistance. Cardiovascular disease is the principal cause of morbidity and premature mortality in people with Type 2 diabetes [1]. The prevalence of the cardiovascular risk factors that comprise the insulin resistance syndrome is increased in Type 2 diabetes [2].

Type 2 diabetes is a complex trait with lifestyle, metabolic and genetic factors contributing to the pathogenesis of the disease. Heritability estimates derived from families with increased susceptibility to Type 2 diabetes provide support for the role of genetic and shared lifestyle factors. As might be expected, several studies of such families from different ethnic groups have shown high heritabilities for measures of glucose tolerance, insulin secretion and insulin action that characterise the diabetic state $[3,4,5]$.

We and others have previously reported an increased prevalence of the features of the insulin resistance syndrome in non-diabetic members of Type 2 diabetic families [6,7]. A study of Type 2 diabetic families of Amish extraction reported high heritabilities for the same cardiovascular risk factors [3]. These studies provide support for a familial predisposition to an increased cardiovascular risk as well as to diabetes in families with increased susceptibility to Type 2 diabetes. Interestingly, high heritability estimates for the same traits have also recently been reported for UK families of northern European origin that were otherwise healthy and had no predisposition to Type 2 diabetes [8].

The aim of this study was to measure the heritability estimates for metabolic traits and features of the insulin resistance syndrome in families with increased susceptibility to Type 2 diabetes.

\section{Subjects and methods}

Subject recruitment. This study is based upon the Diabetes UK Warren Type 2 Diabetes Repository. A collection of 843 pedigrees with at least two Type 2 diabetic sibs was established by six UK diabetes research centres between 1995 and 1998. The details of the collection have already been described [9], but a key feature was that all families were of northern European extraction defined as all four grandparents of the affected sibs being of British and/or Irish origin.

The collection was then extended by the recruitment of non-diabetic sibs and offspring of the original Type 2 diabetic family members. All unaffected subjects had to be of good general health and aged between 35 and 80 years for sibs and over 18 years for offspring. For the offspring, the non-diabetic spouse of the original Type 2 diabetic parent had also to be of British and/or Irish origin. Patients having long-term steroid therapy and women pregnant at the time of recruitment were excluded. A key objective was to target recruitment towards unaffected offspring pairs for future quantitative trait analysis. Figure 1 shows a representative pedigree.

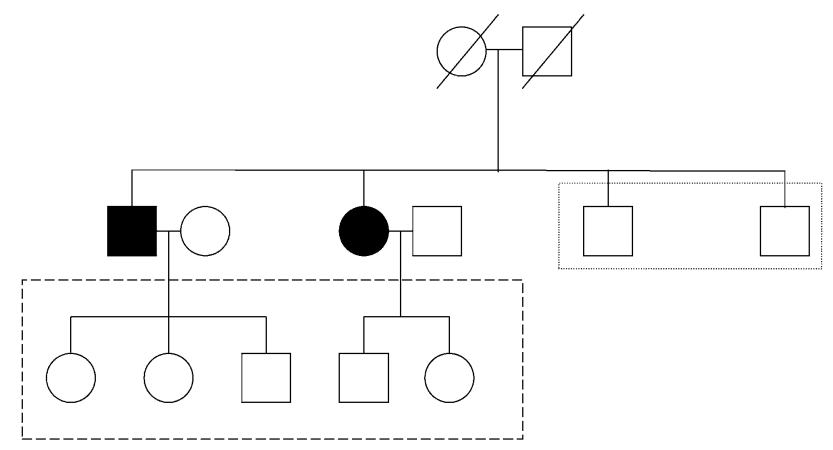

Fig. 1. A representative pedigree showing the affected Type 2 diabetic sib-pair (filled symbols) and unaffected siblings (empty symbols within dotted box) and unaffected offspring (empty symbols within dashed box) suitable for recruitment

All subjects gave written informed consent and the project protocols were approved by the local research ethics committees.

Subject characterisation. Measurements were taken according to a common protocol across the six recruitment centres and techniques standardised at a pre-recruitment training day. Anthropometric measurements (height, weight, BMI, waist circumference, hip circumference and WHR) were recorded and blood for DNA was sampled from all recruited family members, i.e. the Type 2 diabetic patients and the non-diabetic sibs and offspring.

The non-diabetic subjects also underwent more detailed characterisation. They were asked to fast from 22.00 hours the night before assessment, and to refrain from excessive exercise and alcohol for the previous $24 \mathrm{~h}$. Subjects underwent a shortened OGTT to assess the early insulin response (EIR). An indwelling cannula was introduced into an antecubital vein. After $15 \mathrm{~min}$, three fasting blood samples were removed at 5-min intervals prior to an oral glucose load. At $0 \mathrm{~min}$, the subject was asked to take a 75-g oral glucose load and blood was sampled at 10, 25 and $30 \mathrm{~min}$. The cannula was then removed and the subject given breakfast. Of the 811 relatives, 132 declined the oral glucose load, and so the 30 -min glucose concentration and the EIR were not available for these subjects.

Body composition was assessed by bioelectrical impedance (Bodystat 1500, Douglas, Isle of Man, British Isles). Blood pressure was recorded (3 times at 2-min intervals) using an Omron 705CP (Vernon Hills, Ill., USA) after the subject had been seated for $5 \mathrm{~min}$.

Sample analysis. Except for plasma glucose, all samples were analysed at a central laboratory in Newcastle $\left(\mathrm{HbA}_{1} \mathrm{c}\right.$ was analysed in Exeter). Plasma glucose concentrations were measured locally by the glucose oxidase method, and each centre participated in a national quality control programme for glucose measurements. We measured $\mathrm{HbA}_{1} \mathrm{c}$ using an HPLC-based method. Serum insulin, total proinsulin and C-peptide concentrations were measured by specific enzyme immunoassays (Dako Diagnostics, Ely, UK), and serum leptin by radio-immunoassay (Linco, Biogenesis, Poole, UK). Plasma non-esterified fatty acid concentrations were measured by centrifugal enzymatic analysis (Wako, Neuss, Germany). Serum total cholesterol and triglyceride concentrations were measured by cholesterol oxidase-peroxidase and lipase-glycerol kinase methods respectively using commercial kits (Boehringer Mannheim, Lewes, $\mathrm{UK})$. We measured the HDL cholesterol by assaying the supernatant cholesterol concentration after precipitation of apolipo- 
protein-B-containing lipoproteins, and LDL cholesterol concentration was calculated using the Friedewald formula [10].

Data analysis. Homeostasis model assessment of pancreatic beta cell function (HOMA \%B) and of insulin sensitivity (HOMA \%S) were derived using the HOMA program [11] provided by J.C. Levy. We calculated the EIR using the formula (30-min insulin-mean fasting insulin)/30-min glucose [12].

Data were prepared and initially analysed using the software Minitab. Plots were produced using centres, sex and relationship (sibling or offspring) as factors to better understand the variability within the data. Log transformations were produced for all measurements (except for body composition and blood pressure) to normalise distributions. A regression analysis was performed to identify important predictors used to select covariates. The transformed data were used to carry out polygenic analysis using the software package SOLAR (Southwest Foundation for Biomedical Research, San Antonio, Tex., USA). With this package, maximum likelihood estimation was used to fit mixed models with fixed covariate effects, additive genetic effects and residual error. Heritability estimates (with their standard errors) were derived for each variable of interest for various choices of covariates. Heritability denotes the proportion of phenotypic variance, after accounting for covariates, explained by additive genetic effects. However, there is a possibility of an upward bias due to common environmental effects. Initially, the sibs and offspring were analysed separately. However, as there was no evidence of a difference between the heritability estimates for any of the variables of interest, only the combined estimates using information from multi-generation and single-generation groups are presented here.

Freeman and colleagues [8] derived heritability estimates for key features of the insulin resistance syndrome in healthy families of northern European origin in which the proband had no clinical evidence of cardiovascular disease or diabetes. For traits common to our study, we transformed and analysed our data in the same way using the SOLAR package and adjusted for the same covariates (age and sex). We then compared heritability estimates for each trait between the two studies and considered differences to be statistically significant when the estimates differed by four times the SED $(p<0.001)$.

\section{Results}

Study population. Of the 845 recruited siblings or offspring of the original Type 2 diabetic sib-pair, 34 were found to have diabetes at the time of investigation and were excluded from further analysis. This left 811 non-diabetic relatives recruited from 278 pedigrees. Table 1 summarises the distribution of non-diabetic relatives across the families and yields an average pedigree size of just less than three non-diabetic relatives per family. Tables 2 and 3 break this information down into offspring and siblings respectively. It can be seen that the majority of recruited relatives were offspring. In addition, families with two offspring were the most common (Table 2), reflecting the recruitment strategy, which was to select for offspring pairs.

Table 4 summarises the clinical characteristics of the subjects, with the offspring and sibling data pre-
Table 1. Pedigree structure: non-diabetic relatives (offspring and siblings) per family

\begin{tabular}{lll}
\hline $\begin{array}{l}\text { Number of } \\
\text { relatives } \\
\text { per family }\end{array}$ & $\begin{array}{l}\text { Number of } \\
\text { families } \\
(n=278)\end{array}$ & $\begin{array}{l}\text { Number of } \\
\text { individuals } \\
(n=811)\end{array}$ \\
\hline 1 & 50 & 50 \\
2 & 96 & 192 \\
3 & 57 & 171 \\
4 & 35 & 140 \\
5 & 14 & 70 \\
6 & 11 & 66 \\
7 & 6 & 42 \\
8 & 2 & 16 \\
9 & 6 & 54 \\
10 & 1 & 10 \\
\hline
\end{tabular}

Table 2. Distribution of offspring by family

\begin{tabular}{lcc}
\hline $\begin{array}{l}\text { Number of } \\
\text { offspring } \\
\text { per family }\end{array}$ & $\begin{array}{l}\text { Number of } \\
\text { families } \\
(n=278)\end{array}$ & $\begin{array}{l}\text { Number of } \\
\text { individuals } \\
(n=586)\end{array}$ \\
\hline 0 & 69 & 0 \\
1 & 9 & 9 \\
2 & 109 & 218 \\
3 & 42 & 126 \\
4 & 28 & 112 \\
5 & 10 & 50 \\
6 & 6 & 36 \\
7 & 5 & 35 \\
\hline
\end{tabular}

Table 3. Distribution of siblings by family

\begin{tabular}{lll}
\hline $\begin{array}{l}\text { Number of } \\
\text { siblings } \\
\text { per family }\end{array}$ & $\begin{array}{l}\text { Number of } \\
\text { families } \\
(n=278)\end{array}$ & $\begin{array}{l}\text { Number } \\
\text { of individuals } \\
(n=225)\end{array}$ \\
\hline 0 & 160 & 0 \\
1 & 60 & 60 \\
2 & 33 & 66 \\
3 & 14 & 42 \\
4 & 4 & 16 \\
5 & 3 & 15 \\
6 & 3 & 18 \\
7 & 0 & 0 \\
8 & 1 & 8 \\
\hline
\end{tabular}

sented separately. The siblings were, on average, around 20 years older than the offspring. The expected differences in percentage body fat, WHR, serum leptin and HDL cholesterol were seen between the sexes in both the siblings and the offspring. In addition, for both males and females there were differences between offspring and siblings for percentage body fat, fasting glucose, HOMA $\% \mathrm{~B}$, total cholesterol and blood pressure that are likely to reflect age-related changes. 
Table 4. Clinical characteristics for non-diabetic offspring and siblings

\begin{tabular}{|c|c|c|c|c|c|c|c|c|}
\hline \multirow[t]{2}{*}{ Characteristic } & \multicolumn{4}{|c|}{ Offspring } & \multicolumn{4}{|c|}{ Siblings } \\
\hline & \multicolumn{2}{|c|}{ Women $(n=313)$} & \multicolumn{2}{|c|}{ Men $(n=273)$} & \multicolumn{2}{|c|}{ Women $(n=128)$} & \multicolumn{2}{|c|}{$\operatorname{Men}(n=97)$} \\
\hline BMI $\left(\mathrm{kg} / \mathrm{m}^{2}\right)$ & 28.08 & 6.24 & 27.36 & 4.44 & 28.07 & 4.73 & 27.45 & 5.03 \\
\hline WHR & 0.81 & 0.08 & 0.92 & 0.06 & 0.84 & 0.06 & 0.95 & 0.06 \\
\hline 30-min glucose $(\mathrm{mmol} / \mathrm{l})$ & 7.67 & 1.51 & 8.85 & 1.60 & 9.12 & 1.62 & 9.42 & 1.46 \\
\hline $\mathrm{HbA}_{1} \mathrm{c}(\%)$ & 4.72 & 0.38 & 4.77 & 0.42 & 5.12 & 0.50 & 5.12 & 0.46 \\
\hline Age (years) & 39.41 & 7.79 & 38.55 & 7.51 & 63.49 & 8.73 & 62.64 & 10.11 \\
\hline Fasting insulin (mU/l) & 8.89 & 5.43 & 9.93 & 5.55 & 9.05 & 4.64 & 10.02 & 5.83 \\
\hline Fasting pro-insulin (pmol/l) & 5.66 & 3.59 & 7.35 & 5.71 & 7.19 & 5.40 & 8.26 & 5.28 \\
\hline Fasting C-peptide (nmol/l) & 0.62 & 0.26 & 0.68 & 0.29 & 0.68 & 0.27 & 0.76 & 0.37 \\
\hline LDL cholesterol (mmol/l) & 3.40 & 1.00 & 3.62 & 0.93 & 4.16 & 0.96 & 4.06 & 1.17 \\
\hline $\mathrm{NEFA}(\mathrm{mmol} / \mathrm{l})$ & 0.53 & 0.20 & 0.43 & 0.19 & 0.63 & 0.19 & 0.50 & 0.20 \\
\hline HOMA \%B & 94.19 & 36.94 & 92.99 & 37.88 & 82.51 & 30.86 & 83.22 & 31.34 \\
\hline HOMA \%S & 130.00 & 65.78 & 114.39 & 61.28 & 118.32 & 52.76 & 113.41 & 58.92 \\
\hline Systolic BP (mmHg) & 116.15 & 13.94 & 127.92 & 13.00 & 136.93 & 18.72 & 142.59 & 20.90 \\
\hline Diastolic BP (mmHg) & 73.56 & 9.34 & 78.90 & 8.67 & 77.76 & 10.49 & 81.81 & 10.03 \\
\hline EIR (mU/mmol) & 5.71 & 3.46 & 6.00 & 4.30 & 4.38 & 3.18 & 5.05 & 3.85 \\
\hline
\end{tabular}

NEFA, non-esterified fatty acids; HOMA \%B, homeostasis model assessment of beta cell function; HOMA \%S, homeostasis model assessment of insulin sensitivity; EIR, early insulin response

Table 5. Heritability estimates for metabolic and anthropometric characteristics, unadjusted (Model 1) and after correction for age and sex (Model 2), for age, sex and BMI (Model 3) and for age, sex, BMI and recruitment centre (Model 4)

\begin{tabular}{|c|c|c|c|c|c|c|c|c|c|c|c|}
\hline \multirow[t]{2}{*}{ Characteristic } & \multicolumn{2}{|c|}{ Model 1} & \multicolumn{3}{|c|}{ Model 2} & \multicolumn{3}{|c|}{ Model 3} & \multicolumn{3}{|c|}{ Model 4} \\
\hline & $\begin{array}{l}\mathrm{h}^{2} \\
\text { mean }\end{array}$ & $\begin{array}{l}\mathrm{h}^{2} \\
\mathrm{SEM}\end{array}$ & $\begin{array}{l}\mathrm{h}^{2} \\
\text { mean }\end{array}$ & $\begin{array}{l}\mathrm{h}^{2} \\
\text { SEM }\end{array}$ & $\begin{array}{l}\text { Covariates } \\
\%\end{array}$ & $\begin{array}{l}\mathrm{h}^{2} \\
\text { mean }\end{array}$ & $\begin{array}{l}\mathrm{h}^{2} \\
\mathrm{SEM}\end{array}$ & $\begin{array}{l}\text { Covariates } \\
\%\end{array}$ & $\begin{array}{l}\mathrm{h}^{2} \\
\text { mean }\end{array}$ & $\begin{array}{l}\mathrm{h}^{2} \\
\text { SEM }\end{array}$ & $\begin{array}{l}\text { Covariates } \\
\%\end{array}$ \\
\hline BMI & 0.54 & $(0.06)$ & 0.52 & $(0.09)$ & 0.48 & & & & & & \\
\hline WHR & 0.19 & $(0.05)$ & 0.31 & $(0.08)$ & 40.8 & 0.22 & $(0.08)$ & 52.2 & 0.22 & $(0.08)$ & 52.0 \\
\hline Body fat & 0.41 & $(0.09)$ & 0.57 & $(0.1)$ & 54.7 & 0.57 & $(0.1)$ & 85.0 & 0.57 & $(0.1)$ & 85.1 \\
\hline $\mathrm{HbA}_{1} \mathrm{c}$ & 0.67 & $(0.1)$ & 0.48 & $(0.09)$ & 18.1 & 0.52 & (0.09) & 18.8 & 0.55 & $(0.09)$ & 20.1 \\
\hline Fasting insulin & 0.30 & $(0.1)$ & 0.30 & $(0.1)$ & 1.3 & 0.43 & $(0.1)$ & 37.8 & 0.37 & $(0.1)$ & 38.2 \\
\hline Fasting pro-insulin & 0.32 & $(0.1)$ & 0.30 & $(0.1)$ & 4.8 & 0.49 & $(0.1)$ & 25.9 & 0.45 & $(0.1)$ & 20.0 \\
\hline Fasting C-peptide & 0.35 & (0.09) & 0.35 & $(0.09)$ & 2.1 & 0.29 & (0.09) & 25.1 & 0.12 & $(0.09)$ & 28.1 \\
\hline Leptin & 0.33 & $(0.1)$ & 0.44 & $(0.1)$ & 39.2 & 0.28 & $(0.1)$ & 70.5 & 0.29 & $(0.1)$ & 62.5 \\
\hline Cholesterol & 0.75 & $(0.1)$ & 0.59 & $(0.1)$ & 15.6 & 0.62 & $(0.1)$ & 16.1 & 0.59 & $(0.1)$ & 16.0 \\
\hline HOMA \%B & 0.69 & $(0.1)$ & 0.67 & $(0.1)$ & 2.4 & 0.80 & (0.09) & 20.2 & 0.78 & $(0.09)$ & 22.9 \\
\hline HOMA \%S & 0.29 & $(0.1)$ & 0.29 & $(0.1)$ & 1.5 & 0.42 & $(0.1)$ & 37.7 & 0.46 & $(0.1)$ & 30.2 \\
\hline Systolic BP & 0.49 & $(0.1)$ & 0.29 & $(0.1)$ & 32.4 & 0.30 & $(0.1)$ & 35.1 & 0.29 & $(0.1)$ & 35.2 \\
\hline Diastolic BP & 0.26 & $(0.09)$ & 0.30 & $(0.09)$ & 11.2 & 0.29 & $(0.09)$ & 15.9 & 0.28 & $(0.09)$ & 15.5 \\
\hline EIR & 0.45 & $(0.1)$ & 0.40 & $(0.1)$ & 4.2 & 0.44 & $(0.1)$ & 12.1 & 0.45 & $(0.1)$ & 15.0 \\
\hline
\end{tabular}

$\mathrm{h}^{2}$, heritability estimate; NEFA, non-esterified fatty acids; HOMA \% B, homeostasis model assessment of beta cell function; HOMA \% , homeostasis model assessment of insulin sensitivity; EIR, early insulin response 
Table 6. Comparison of the heritability estimates [mean (SEM)] for features of the insulin resistance syndrome measured in this study and that of Freeman et al. [8] after adjustment for age and sex

\begin{tabular}{|c|c|c|c|c|c|c|}
\hline \multirow[t]{2}{*}{ Feature } & \multicolumn{2}{|c|}{ This study } & \multicolumn{2}{|c|}{ Freeman et al. } & \multirow[t]{2}{*}{ Difference } & \multirow[t]{2}{*}{ SE of difference } \\
\hline & $h^{2}$ & & $\mathrm{~h}^{2}$ & & & \\
\hline WHR & 0.31 & $(0.08)$ & 0.23 & $(0.11)$ & 0.08 & 0.14 \\
\hline Fasting glucose & 0.77 & $(0.09)$ & 0.21 & $(0.08)$ & $0.56 *$ & 0.12 \\
\hline Fasting insulin & 0.30 & $(0.1)$ & 0.29 & $(0.08)$ & 0.01 & 0.13 \\
\hline Log LDL cholesterol & 0.57 & $(0.1)$ & 0.33 & $(0.09)$ & 0.24 & 0.13 \\
\hline HOMA $\% \mathrm{~S}$ & 0.29 & $(0.1)$ & 0.31 & $(0.08)$ & -0.02 & 0.13 \\
\hline Systolic BP & 0.28 & $(0.1)$ & 0.19 & $(0.08)$ & 0.09 & 0.13 \\
\hline
\end{tabular}

$\mathrm{h}^{2}$, heritability estimate; HOMA $\% \mathrm{~S}$, homeostasis model assessment of insulin sensitivity; ${ }^{*} p<0.001$

Heritability estimates. Table 5 summarises the heritability estimates derived from the SOLAR package for four models of analysis: Model 1 shows the unadjusted estimates; Model 2 shows the estimates adjusted for age and sex; Model 3 shows the estimates adjusted for age, sex and BMI; and Model 4 shows the estimates adjusted for age, sex, BMI and recruitment centre. The percentage contribution of the covariates to the variation in each variable is shown for Models 2, 3 and 4 . The heritability estimates for all variables in each model were statistically significant compared with zero, with $p$ values ranging between 0.02 and $10^{-}$ 6. After correction for the key covariates (Model 4), HOMA $\%$ B and fasting glucose concentration had the highest heritability estimates of 0.78 and 0.72 respectively. Other variables with particularly high adjusted heritability estimates $(>0.50)$ included BMI, percentage body fat, $\mathrm{HbA}_{1} \mathrm{c}$ and fasting lipids (triglycerides, total, LDL and HDL cholesterol). Significant centre effects $(p<0.01)$ under Model 4 were observed for just three variables (HOMA \% B, fasting glucose and fasting C-peptide).

Table 6 compares the heritability estimates for the traits common to our study and a study by Freeman and colleagues [8] after correction for the same covariates (age and sex). The only significant difference between the two studies was for fasting glucose, where the heritability estimates differed by 4 times the SED $(p<0.001)$. However, heritability estimates for other traits (BMI, triglycerides and LDL cholesterol) also tended to be higher in our study, but this did not reach statistical significance based upon this analysis.

A sub-study was conducted to determine whether concurrent medication with anti-hypertensive and/or lipid-lowering therapy influenced heritability estimates. Heritability estimates were derived for 414 individuals from three centres of which 26 were on antihypertensive agents alone, five were on lipid lowering drugs alone, and seven were taking both classes of agents. There were no major differences in the esti- mates of heritability for the variables listed in Table 5 when the 38 patients taking these medications were excluded from the analysis. Considering only those traits known to be influenced by these medications, the heritability estimates (before and after exclusion respectively) were as follows: systolic BP (0.32 and 0.27 ), diastolic BP (0.41 and 0.37), LDL cholesterol (0.54 and 0.56), HDL cholesterol (0.66 and 0.62) and triglycerides (0.43 and 0.45$)$.

\section{Discussion}

This study describes heritability estimates for a wide range of metabolic and anthropometric traits derived from families of northern European extraction with increased susceptibility to Type 2 diabetes. A key finding is the very high heritability estimate for the fasting glucose concentration (Table 5) before and after correction for key covariates (Models 2, 3 and 4). Other studies of similar Type 2 diabetic families have also reported significant but lower heritability estimates for fasting glucose, ranging between 0.10 and $0.63[3,5]$. However, in these studies, fasting glucose measurements for diabetic patients (some of whom were on medication) were included, which as previously considered [5] might affect the variance of the measurement and in turn the heritability estimate. This was not an issue in the present study, in which fasting glucose was measured only in the non-diabetic family members.

Subjects were recruited across six UK centres. However, significant centre effects under Model 4 were observed for just three variables: HOMA \%B, fasting glucose and fasting C-peptide. Fasting glucose was measured at the individual centres, and differences in measurement technique could possibly explain the centre effect for this variable and HOMA \%B from which it is derived. We measured Cpeptide with the other hormones at a central laborato- 
ry, and results might therefore reflect a real difference between people from different centres and/or local storage conditions. However, it can be seen that these significant centre effects had relatively little impact upon the estimates of heritability for these variables. Specifically, fasting glucose and HOMA \%B had the highest heritability estimates at each level of adjustment (Models 2, 3 and 4).

Along with fasting insulin, fasting glucose concentration is a key measurement used by HOMA to derive indices of beta cell function (HOMA \%B) and insulin sensitivity (HOMA \%S). The heritability estimates for both HOMA \%B and HOMA \%S were statistically significant (Table 5). However, the estimate for HOMA \%B (0.78) was higher than that for HOMA $\% \mathrm{~S}$ (0.46) after adjustment for key covariates under Model 4. This is consistent with previous family [5] and twin [13] studies, which have used more direct methods to assess insulin secretion and sensitivity. Watanabe and colleagues [5] studied the non-diabetic members of their Type 2 diabetic family pedigrees using the frequently sampled intravenous glucose tolerance test and reported a higher heritability estimate for insulin secretion than that for insulin sensitivity. Similarly, the heritability estimates for first- and late-phase insulin secretion ( 0.55 and 0.58 respectively) assessed by intravenous glucose tolerance test were higher than that for insulin sensitivity (0.37) assessed by euglycaemic clamp in a study of twin pairs [13]. These observations underline the importance of inherited determinants of insulin secretion, which in turn is recognised as a critical determinant of fasting glucose levels [14] and of diabetes risk [15, 16]. The EIR following an oral glucose load describes another aspect of beta cell function and the heritability estimate for this was also significant under Model 4.

Freeman and colleagues previously reported that the heritability estimates of the key features of the insulin resistance syndrome were high in otherwise healthy families of northern European extraction [8]. One of the aims of our study was to compare the heritabilities of the same traits as estimated in both studies, given that the families in each study had been recruited from the same background population. As shown in Table 6, there was a clear and marked difference between the heritability estimates for fasting glucose. This is consistent with the fact that the studies had different strategies, recruiting families with or without increased susceptibility to Type 2 diabetes. Such clear differences were not apparent for the other traits listed in Table 6, although the heritability estimates for several traits (BMI, fasting triglycerides and LDL cholesterol) also tended to be higher in our study. Interestingly, the heritability estimates for HOMA \%S were comparable between the two studies, indicating that the heritability of insulin sensitivity is not diabetes specific.

In conclusion, families with increased susceptibility to Type 2 diabetes show high heritability estimates for fasting glucose concentration and pancreatic beta cell function. The high heritability estimate for pancreatic beta cell function suggests that this resource may be most effective when investigating genetic susceptibility to beta cell dysfunction. There was also evidence of high heritability estimates for the features of the insulin resistance syndrome in our families, which were similar to those found in subjects from families without diabetes. This supports the notion that insulin resistance syndrome is the "common soil" for a number of metabolic phenotypes including Type 2 diabetes.

Acknowledgements. This study was supported by the Eli Lilly Company and the British Diabetic Association Research Fund. Special thanks to Pat Shearing for technical support and to the study nurses Catherine Turner, Jean Gerrard, Henrietta Mulnier, Barbara Millauer, Gillian Hood, Magie Opomu, Sue Ayres, Nuala Walravens, Beryl Barrow and Kerrie Self.

\section{References}

1. Panzram G (1987) Mortality and survival in type 2 (noninsulin-dependent) diabetes mellitus. Diabetologia 30:123131

2. Hanefield M, Schmechel H, Schwanebeck U, Linder J (1997) Predictors of coronary heart disease and death in NIDDM: the Diabetes Intervention Study experience. Diabetologia 40 [Suppl 2]:S123-S124

3. Hsueh WC, Mitchell BD, Aburomia R et al. (2000) Diabetes in the old order Amish. Characterization and heritability analysis of the Amish Family Diabetes Study. Diabetes Care 23:595-601

4. Sakul H, Pratley R, Cardon L, Ravussin E, Mott D, Bogardus C (1997) Familiarity of physical and metabolic characteristics that predict the development of non-insulindependent diabetes in Pima Indians. Am J Hum Genet 60:651-656

5. Watanabe RM, Valle T, Hauser ER et al. (1999) Familiarity of quantitative metabolic traits in Finnish families with non-insulin-dependent diabetes. Hum Hered 49: 159-168

6. Stewart MW, Humphriss DB, Berrish TS et al. (1995) Features of Syndrome $X$ in first degree relatives of NIDDM patients. Diabetes Care 18:1020-1022

7. Laws A, Stefanick ML, Reaven GM (1989) Insulin resistance and hypertriglyceridaemia in nondiabetic relatives of patients with non-insulin dependent diabetes. J Clin Endocrinol Metab 69:343-347

8. Freeman MS, Mansfield MW, Barrett JH, Grant PJ (2002) Heritability of features of the insulin resistance syndrome in a community-based study of healthy families. Diabetic Med 19:994-999

9. Wiltshire S, Hattersley AT, Hitman GA et al. (2001) A genomewide scan for loci predisposing to type 2 diabetes in a UK population (the Diabetes UK Warren 2 Repository): analysis of 573 pedigrees provides independent replication of a susceptibility locus on chromosome 1q. Am J Hum Genet 69:553-569

10. Friedewald WT, Levy RI, Fredrickson DS (1972) Estimation of the low-density lipoprotein cholesterol in plasma without use of the preparative ultracentrifuge. Clinical Chemistry 18:499-502 
11. Matthews DR, Hosker JP, Rudenski AS, Naylor BA, Treacher DF, Turner RC (1985) Homeostasis model assessment: insulin resistance and B-cell function from fasting plasma glucose and insulin concentrations in man. Diabetologia 28:412-419

12. Wareham NJ, Phillips DI, Byrne CD, Hales CN (1995) The 30 minute insulin incremental response in an oral glucose tolerance test as a measure of insulin secretion. Diabetic Med 12:931

13. Lehtovirta M, Kaprio J, Forsblom C, Eriksson J, Tuomilehto J, Groop L (2000) Insulin sensitivity and insulin secre- tion in monozygotic and dizygotic twins. Diabetologia 43:285-293

14. Porte D Jr (1991) Banting lecture 1990. B-cells in type 2 diabetes mellitus. Diabetes 40:166-180

15. Haffner SM, Miettinen H, Gaskill SP, Stern MP (1995) Decreased insulin secretion and increased insulin resistance are independently related to the 7 year risk of NIDDM in Mexican-Americans. Diabetes 44:1386-1391

16. Tripathy D, Carlsson M, Almgren P et al. (2000) Insulin secretion and insulin sensitivity in relation to glucose tolerance. Diabetes 49:975-980 